\title{
Contemporary management strategies of blunt tracheobronchial injuries
}

\author{
Anna Rieth ${ }^{\mathrm{a}, *}$, Endre Varga ${ }^{\mathrm{b}}$, Tamás Kovács ${ }^{\mathrm{a}}$, Aurél Ottlakán ${ }^{\mathrm{c}}$, Tibor Németh ${ }^{\mathrm{c}}$, József Furák ${ }^{\mathrm{c}}$ \\ a Division of Pediatric Surgery, Department of Pediatrics, University of Szeged, Szeged, Hungary \\ ${ }^{\mathrm{b}}$ Department of Traumatology, University of Szeged, Szeged, Hungary \\ ${ }^{\mathrm{c}}$ Division of Thoracic Surgery, Department of Surgery, University of Szeged, Szeged, Hungary
}

\section{A R T I C L E I N $\mathrm{N}$ F $\mathrm{O}$}

\section{Article history:}

Accepted 9 July 2020

Available online xxx

\section{Keywords:}

Tracheobronchial injury

Airway management

Bronchus

Rupture

Blunt thoracic trauma

Bronchial injury

\begin{abstract}
A B S T R A C T
Background: Tracheobronchial injuries are rare but feasibly life-threatening conditions. A prompt diagnosis and early management can be lifesaving. Due to the unspecific symptoms and indirect radiological signs the diagnosis often delays.

Objectives: We present a short series of patients suffering from tracheobronchial airway laceration. All the three patients had blunt thoracic or neck trauma and showed early signs of tracheobronchial injury. In the first case a 44-year-old woman was crushed by a bus. Subcutaneous emphysema, pneumothorax on chest computed tomography and hypoxaemia despite of chest tube suggested the presence of an airway injury. During operation a 4-cm-long tear of the trachea and a complete transection of the right main bronchus were found. In the second case a 12-year-old girl was crossed by a truck trailer. Early signs were respiratory failure, extended subcutaneous emphysema, blood clot in the larynx, pneumothorax on both sides. Chest CT showed pneumomediastinum. During the operation a longitudinal laceration was found separating the two main bronchi at the bifurcation. In the third case a 9-year-old boy was injured in a car accident, when the seat-belt crossed his neck. Spreading subcutaneous emphysema, pneumomediastinum and an overinflated endotracheal tube's cuff were found on CT. A completely transected trachea between the first and second tracheal rings was found. All three patients required fast intubation and bronchoscopic examination to confirm the diagnosis, and to identify the site of lacerations. All the patients underwent primary reconstruction and recovered successfully.

Conclusions: In case of suspected tracheobronchial injury, a high index of suspicion is required for early diagnosis. Most commonly respiratory distress, subcutaneous emphysema and pneumothorax are found on physical examination. Prompt intubation below the site of the injury and early laryngo- or bronchoscopic examination have priority, as we did in our cases. A primary anastomosis is required with minimal resection during urgent operation. A better outcome is to be expected when extubation is done early after surgery. We offer ordinal steps that should be taken to lead to a prompt management and good long-term outcome based on the literature and our experiences.
\end{abstract}

(c) 2020 Published by Elsevier Ltd.

\section{Introduction}

Tracheobronchial injury (TBI) is an uncommon but can become a life-threatening condition, and the majority of these patients (30-80\%) still die at the scene of the accident [1,2]. Post-mortem analyses show a $2,8 \%$ incidence of TBI after blunt trauma described

\footnotetext{
This paper is part of a supplement supported by the Hungarian Trauma Society.

* Corresponding author: 14-15. Korányi Avenue, Szeged, Hungary 6725.

E-mail addresses: rieth.anna@med.u-szeged.hu (A. Rieth), office.trauma@med.uszeged.hu (E. Varga), kovacs.tamas@med.u-szeged.hu (T. Kovács), ottlakan.aurel@med.u-szeged.hu (A. Ottlakán), nemeth.tibor@med.u-szeged.hu (T. Németh), jfurak@gmail.com (J. Furák).
}

by Bertelsen and Howitz [3]. Amongst trauma patients suffering neck or chest injury who reached emergency department, TBI incidence is estimated at $0,2-8 \%[1,4,5]$. The incidence of TBI amongst children is rarer than in adults, $<0,05 \%$ of chest traumas cause airway injury, and the associated mortality is 8,7\% [6,7]. Early diagnosis is essential for patient survival. Avoidance of airway obstruction and early, successful surgical management are vital for a successful outcome. The lack of typical clinical symptoms and the various appearance of radiological signs often delay the diagnosis. For successful treatment we should keep the possibility of an airway injury in mind, and we should be aware of the symptoms and management. With the presentation of our three cases, we discuss the 
diagnosis and the treatment of the TBI located on the neck and around the bifurcation.

\section{Case series}

Case 1

A 44-year-old woman was referred to our hospital, who had been found under a bus in an accident. After being rescued she was hypoxic despite oxygen therapy, and subcutaneous emphysema was found in the jugulum. In the nearest county hospital primary survey according to ATLS (Advanced Trauma Life Support) [8] protocol was applied and computed tomography (CT) of the head, neck, thorax and abdomen and CT angiography were performed. The following injury complex were revealed: left-sided pulmonary contusion, right-sided pneumothorax, associated with the occlusion of the right subclavian artery and multiple rib fractures on both sides. The patient was still hypoxic despite of chest decompression on the right side. At this point, airway injury was expected and a double-lumen endotracheal tube (DLT) was inserted to guarantee isolated, one-lung ventilation on left side. Her respiratory parameters hardly improved (SpO2 80\% with 100\% oxygen therapy), and she was transferred to our department for further investigations. Upon arrival her circulation was unstable and required noradrenalin support, her blood pressure was high $(150 / 90 \mathrm{mmHg}$ ) but persistently reducing, and saturation was $93 \%$. Deformed and sunk right side of the bony thorax and diminished breathing sounds over the right lower lobe were detected on physical examination. Bronchoscopic examination revealed a rupture of the right main bronchus at the level of the carina. Urgent right sided posterolateral thoracotomy was performed under doublelumen tube intubation to the left bronchus. A large pneumomediastinum was found, with intact mediastinal pleura. After opening the mediastinal pleura, a $4 \mathrm{~cm}$ tear was discovered on the lower third of the membranous trachea, which was running along to the left main bronchus $1 \mathrm{~cm}$ distal to the bifurcation. In addition, the right main bronchus was almost completely separated from the carina. The rupture of the membranous part of the trachea and the left mainstem bronchus was reconstructed with interrupted 3-0 absorbable sutures, and the right mainstem bronchus was sutured to the carina with interrupted sutures. The anastomosis was covered with a pedicle intercostal muscle flap. The subclavian arterial injury did not require urgent vascular procedure. Her recovery was uneventful. Chest tube was removed on the sixth postoperative day, and on the seventh day she was discharged from intensive care unit for rehabilitation (Figs. 1, 2).

Case 2

A 12-year-old girl was transferred to our hospital after falling down from a truck trailer over $60 \mathrm{~cm}$ high, and the trailer passed across her chest. At the scene of the accident she was in respiratory distress and had subcutaneous emphysema on her neck. Tension pneumothorax was suspected, therefore right-sided decompression by a needle and endotracheal intubation was accomplished at the scene. During intubation blood clot was found in the larynx. On presentation at our hospital an extended emphysema on the neck and thorax, and no breathing sounds over the left lung were detected. During ATLS management chest tubes were inserted into both sides, and her condition became stable (Fig. 3). Computed tomography of the head, neck, thorax and abdomen revealed pulmonary contusion on both sides, subcutaneous emphysema and fracture of collum chirurgicum humeri. In addition, pneumomediastinum caused by a rupture of the bifurcation was suspected. Bronchoscopy showed a deformity in the bifurcation, but its lumen was still intact. Also suspicion of a tear on the posterior wall of the

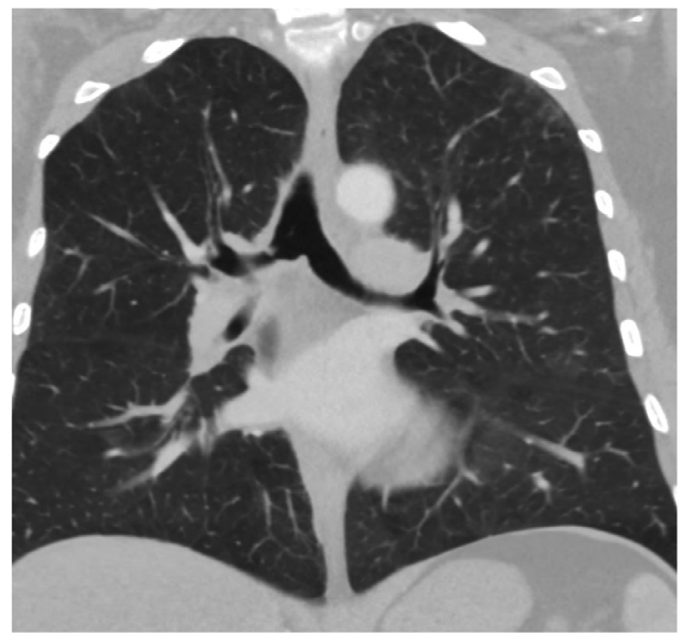

Fig. 1. Control chest CT after half year in Case 1.

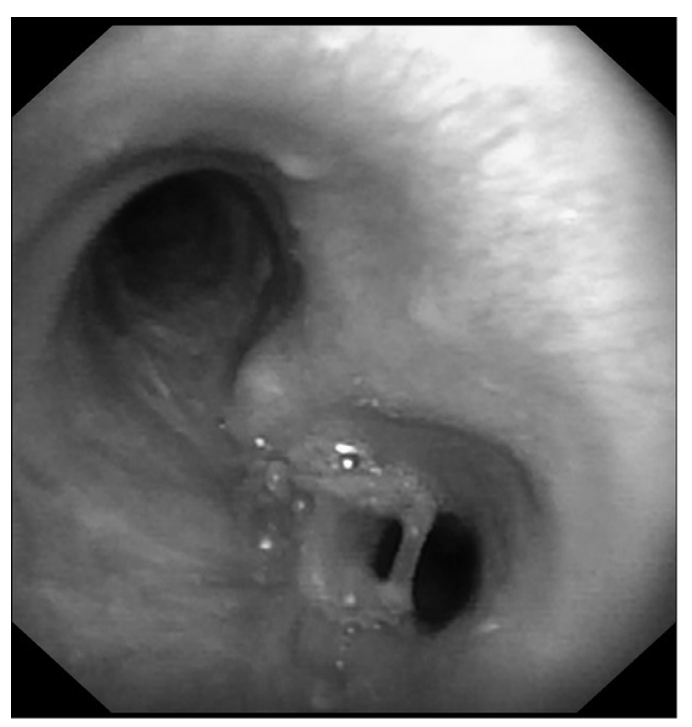

Fig. 2. Bronchoscopy shows the anastomosis after half year in Case 1.

right main bronchus was found. The patient was stable, ventilation and respiratory parameters were appropriate, lungs on both sides were expanded. After two days her parameters did not tend to improve, she still required respiratory support, and a repeated bronchoscopy declared the necessity of surgery. A right sided posterolateral thoracotomy was performed on the third day with single lumen endotracheal tube. A large pneumomediastinum was found with intact mediastinal pleura. Ventilation with single lumen tube was sufficient, but when we opened the mediastinal pleura, all the ventilated air was lost. An urgent exploration was necessary: a longitudinal laceration in the midline of the trachea was running along the bifurcation - a 2-cm-long rupture on pars membranacea and also a 2-cm on pars cartilaginea, - that the two primary bronchi were separated by. After the short exploration, the endotracheal tube was placed into the right and later into the left main bronchus guiding by the operator's finger. A complete reconstruction of the rupture of the carina was performed with interrupted 3-0 absorbable sutures, and the suture line was covered with a pedicle intercostal muscle flap to separate from the oesophagus. After an uneventful recovery chest tubes were removed after 3 days, and she was discharged on the 11th postoperative day. 


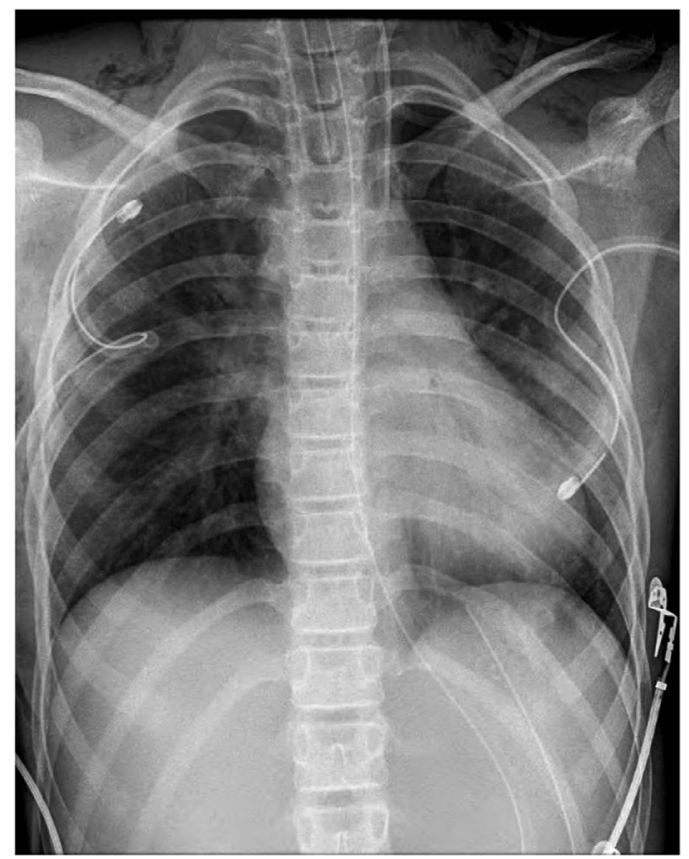

Fig. 3. Preoperative chest film with subcutaneous emphysema in Case 2 .

Case 3

A 9-year old boy was referred to the emergency room after injured as a passenger in a car accident. The car was rolled over and crushed into a tree. At the scene of the accident the child coughed some blood up, soon after he needed resuscitation, which was successful. Intubation and left thoracotomy were applied before transporting. Arriving to our department primary survey began according to ATLS protocol. On physical examination, a contusion sign was found over his neck certainly caused by the safety seat belt. He was stable and his ventilation was satisfying, therefore CT scan was performed. It revealed an extremely spreading subcutaneous emphysema starting from the deep cervical region and travelled through the wall of the thorax and abdomen. A small amount of pneumomediastinum was also discovered, but there was no sign of pneumothorax. It could be seen that the tracheal tube's cuff was over-inflated (Fig. 4). Associated injuries were the followings: severe cerebral oedema and hypoxic brain injury, left tibial fracture and right-sided pulmonary contusion. Based on the history of a direct cervical trauma accompanied by deep cervical emphysema spreading to the abdomen, the possibility of tracheal laceration was considered. Otolaryngologist examined and excluded the presence of tracheal rupture above and below the site of the endotracheal tube's cuff. During surgery a complete transection between the first and second tracheal rings was found, and the ends of the ruptured parts were located $3 \mathrm{~cm}$ apart from each other. The cuff was lying between the two separated tracheal part. The tube's distal half was ended in the lower trachea and provided satisfying ventilation (Fig. 5). Primary closure was done with tensionfree interrupted sutures, and drain was left for two days. By using a chin-to-chest stitch we ensured neck flexion and tension-free anastomosis. Due to the severe brain damage and pulmonary contusion extubation could not be managed in the operating room, and the patient required long-term ventilatory support. Laryngeal oedema and tracheal stenosis developed; thus tracheostomy was done and patient received steroid. Successful extubation was managed on the 11th postoperative day, and tracheostomy cannula was removed after one week, without any sign of stenosis.
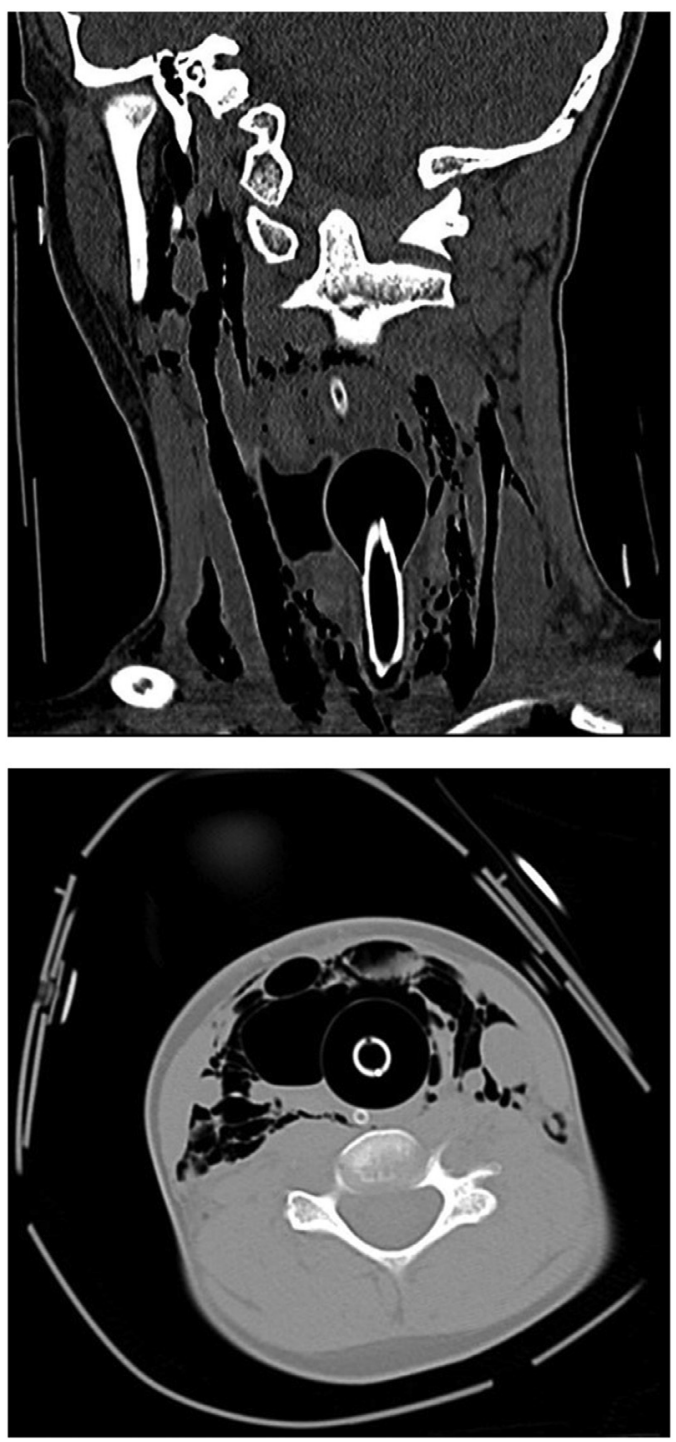

Fig. 4. Overinflated endotracheal tube's cuff and deep cervical emphysema seen on MDCT in Case 3. While the distal part is intact, the proximal part of the trachea cannot be detected.

\section{Discussion}

\section{Epidemiology of TBI}

Injury of tracheobronchial tree occurs by iatrogenic or traumatic cause, which includes blunt or penetrating injury on the neck and thorax. The majority of patients having TBI involve in a highvelocity car or a motor accident. Other injuries include falling from height, crush trauma or pedestrian accidents [5,9]. Blunt trauma is responsible for TBI approximately in $94 \%$ of cases in children, while penetrating injuries are more frequent in adults [6]. All of our cases caused by blunt traumas.

Trachea is more vulnerable at the cervical region. Proximal or middle third tracheal injury commonly appears in penetrating trauma and more likely to be identified and repaired in an early stage. Blunt traumas on neck are mostly occurred due to direct injury or sudden hyperextension. Typical example for direct force is when the trachea is pushed to the oesophagus and the stable vertebral body by an elevated seatbelt, as it was seen in our third case. Based on this mechanism, it can be declared that tracheal rupture tends to be at the exact level of the direct trauma and 

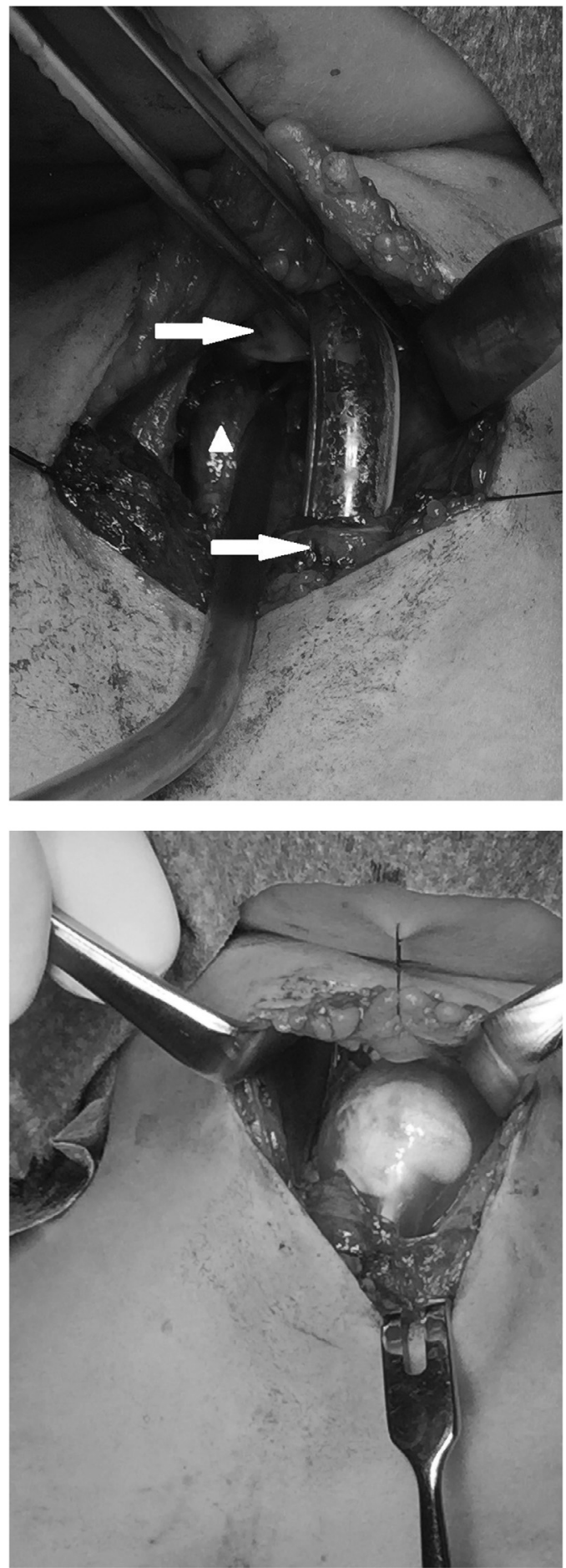

Fig. 5. Intraoperative picture in Case 3. Ruptured tracheal parts are marked by arrows. Triangle shows intact oesophagus.

often associated with oesophageal injury. During compression the sudden increased intratracheal pressure may lead to tracheal tear, paramedian vertical fracture of the larynx or trachea, or even to complete laryngo-tracheal separation. Rapid hyperextension mostly appears when a vehicle is hit from the back, usually following by smashing of the neck into the dash-board, as it is called dashboard tracheal injury.

The paper of Kirsh et al. declared three different types of mechanism causing intrathoracic TBI in blunt traumas [10]. The most common mechanism is sudden anteroposterior compression, when these forces lead to lateral separation in the carina. In the first two cases of ours TBI developed due to the abovementioned mechanism, when the vehicles caused an anteroposterior compression on the chest. The second type includes compression on the chest while the glottis is closed, causing abruptly increased airway pressure. The third way is based on the relative fixation at the level of bifurcation and cricoid cartilage while the lungs are more mobile in the pleural space. In this state an acceleration-deceleration mechanism can cause a shearing force and disruption of the airway. These explain that bronchial injuries mostly occur in motor vehicle accidents, caused by flexion-extension forces.

\section{Location, size and classification of TBI}

Lesions can be distinguished as longitudinal or transversal, and single or complex, when more than one lesions exist. Transverse tears can be partial or complete. In partial tears the airway may remain intact and prevents air-leakage and causes only mild symptoms leading to a delayed diagnosis. A complete airway rupture is generally life-threatening and often associated with severe concomitant injuries.

Upper airway damages can be classified as supraglottic, transglottic or subglottic injuries based on the site of the laceration. In generally the membranous part of the trachea is the weakest point and most likely for disruption, compared with the anterior and lateral wall which are strengthen by the U-shaped tracheal rings. Therefore, the anterior trachea is mostly injured in penetrating injuries, while the posterior wall is mainly affected in blunt traumas [4].

Literature data is consistent, that $75-90 \%$ of TBIs in blunt trauma are located within $2-3 \mathrm{~cm}$ of the carina and $60 \%$ of them are within $1 \mathrm{~cm}[1,2,9,11]$.

The majority of the literature states that the main bronchi are frequently injured (66-85\%), especially on the right side (27-61\%) $[2,9,12,13,14,15]$. Compared with it the cervical trachea (4-19\%), distal thoracic trachea (22\%), left proximal main bronchus (17-32\%) or lobar orifices are less frequently affected (14-16\%) [7,12,13].

The abovementioned right sided predominance is probably known as the right bronchus is more fixed in the thorax, but less protected by surrounding vascular entities than the left one. The right side has also a heavier connecting lung parenchyma on a shorter bronchus [11]. In addition, the right bronchial tree lays close to the thoracic vertebrae, and has the risk to be compressed on the spine during an accident. These anatomy specialities explain that right-sided lesions are more frequent and have higher mortality rate than left-sided. In comparison, the diagnosis tends to be delayed in left-sided cases, due to the abovementioned protective adjacent structures [2].

There are only few data in the literature about morphological classification and related treatment options in TBI patients. For laryngotracheal ruptures Schaefer and Fuhrman defined a system based on the depth of the lesion [14]. In Group I. and II. minor endolaryngeal laceration or mucosal oedema and haematoma appear, and nondisplaced fracture is in Group II. Massive oedema, displaced fracture and vocal cord paralysis defined as Group III. While in Group IV. unstable fracture or more than two fracture lines are present, in Group V. a complete laryngeal separation appears. This study recommends conservative therapy in Group I., tracheostomy in II., and emergent tracheostomy and surgical exploration in Group III., IV. and V.

Another method described by Cardillo et al. declares different levels of laceration and offers a key to decide between conservative and surgical treatment [16]. Level I and II is defined as an incomplete laceration in the layers of tracheal wall. Level III is a complete lesion in all muscular layers of the trachea with herniation of the oesophagus or mediastinal tissue. Cardillo's prospective 
study advises a non-surgical management for Level I, II and III/A, where 29 adult patients with a lesion in these levels were successfully treated with fibrin glue. The presence of oesophageal injury or mediastinitis (Level III/B) determines the need of a prompt surgical treatment. Although this classification method is originally used in postintubation tracheobronchial laceration, some authors promote this method in the management of traumatic airway injuries as well.

\section{Diagnosis}

\section{Symptoms}

Tracheobronchial injuries are difficult to diagnose due to the unspecific symptoms on physical examination and signs in radiology. The presence of concomitant injuries can obscure the symptoms of airway lesion and leads to a delayed diagnosis and early fatal outcome [1]. Therefore, it cannot be overemphasised that a high index of suspicion is the most important clue for prompt diagnosis. TBI has a delayed diagnosis in $25 \%$ to $68 \%$ of patients [11]. Sometimes days or even weeks pass after the trauma until the correct diagnosis is revealed.

The most common symptoms are tachypnoea, respiratory distress (59-100\%), and haemoptysis (14-74\%). Dyspnoea and stridor can be an early sign of tracheal stenosis. Cervical trauma leads to hoarseness or dysphonia causing by laryngeal fracture, vocal cord tears or recurrent laryngeal nerve injury. Nevertheless, subcutaneous emphysema is the classic hallmark (35-87\%) of TBI. Extremely spreading subcutaneous emphysema could even reach the abdomen and lower extremity skin [13]. When a progressive emphysema following blunt trauma occurs, the possibility of TBI should be always considered [1,2,11], as it was in our third case. Spreading subcutaneous emphysema which stops after intubation verifies the diagnosis. We should bear it in mind that emphysema and pneumomediastinum may not appear initially [11]. Even in patients with complete transection, adequate lung ventilation can be managed due to the surrounding tissue which covers the separated fragments and restrain air-leakage.

Lesion in the lower airway causes pneumothorax in $17 \%-72 \%$ of patients, as it was presented in our first two cases. Airway injury generally maintains a massive air-leak through chest drainage and restrains the re-expansion of the lung, which should always alert the clinician to the possibility of TBI $[1,4,11,17]$, as it happened in our first case. On the other hand, a complete transection may lead to occlusion in the airway caused by the surrounding soft tissue, which prevents developing pneumothorax.

Another sign is due to the mediastinal air-filled tissue causing a rasping rumour, called Hamman's sign. It is detected synchronous with the heartbeat on auscultation [1].

Up to $40 \%-100 \%$ of patients with blunt chest trauma are associated with injuries of the abdomen, head or orthopaedic structures [5]. For instance, the most frequent coexistent injuries in cervical region are oesophageal injuries, hemopneumothorax, carotid artery or jugular venous injuries, recurrent laryngeal injuries, cervical spine and larynx injuries. Intrathoracic blunt trauma often involves the great vessels, oesophagus and heart, and causes pulmonary contusion, rib or burst vertebral fracture.

\section{Radiology}

Various indirect signs of an airway lesion can be detected by radiology routinely performed in acute emergency situation; however direct, obvious findings are rarely occurred. Since the introduction of Advanced Trauma Life Support (ATLS) patients with TBI have a lower mortality rate [2]. Primary survey includes eFAST examination (Extended Focused Assessment with Sonography in
Trauma) recommended by ATLS 10th edition [8]. It has a high sensitivity for detecting the presence of pneumo- or hemothorax. The transducer should be placed in the superior pectoral region either on the left and right side, and both $\mathrm{M}$ and $\mathrm{B}$ mode should be applied. Subcutaneous emphysema may add a limitation, but can suggest the presence of tracheobronchial injury.

If endotracheal intubation is successfully managed, but persistent hypoxaemia without pneumothorax is seen on eFAST examination, we should keep an attention of airway injury [17]. On chest $\mathrm{X}$-ray (CXR) the three typical signs of TBI are subcutaneous emphysema, pneumothorax and pneumomediastinum $[1,2,6,12]$. If subcutaneous emphysema is accompanied by the hyoid bone's elevation above $\mathrm{C} 3$ in the neck the possibility of an upper airway transection and rising of the larynx is high. Endotracheal tube presenting external from the airway or an over-inflated endotracheal tube cuff are additional radiologic fidings $[1,9]$. Other indirect signs that may appear is tracheal deformity, discontinuity of the tracheal or bronchial air column. Almost the only direct and pathognomic CXR sign of TBI is the "fallen lung sign". This means that complete transection of the bronchi makes the separated lung to collapse laterally and posteriorly, while in other cases of pneumothorax lung tends to get collapsed to the hilum $[5,9,13]$.

These aforementioned diagnostic tools help the clinicians to suspect airway injury. At this point Bagga et al. defined the criterion that may signify the need of an urgent chest computed tomography: 1) hypoxaemia without pneumothorax after endotracheal intubation, 2) massive air leak after tube thoracotomy and persistent pneumothorax, 3) progressive subcutaneous emphysema and pneumomediastinum [17].

Multi-slice detector computed tomography (MDCT) is superior to traumatic radiographic methods, it can identify and localize the lesion and detect concomitant injuries as well. Unfortunately, direct visualization of tracheobronchial disruption such as complete transection, tracheal ring fracture or fallen lung sign is less reliable. In case of upper airway injury CT scan may identify paratracheal air, deep cervical emphysema or pneumomediastinum. In case of patients with lower airway injury CT can reveal the following findings: pneumomediastinum, mediastinitis, mediastinal haematoma, focal intimal flap in the lumen, bronchial stenosis or obstruction $[5,12,13,17,18]$. Although direct signs are less common, the presence of tracheobronchial interstitial air or gas dispersion around the lesion are remarkable, and should be an alert sign. The sensitivity of CT imaging is seen in up to $71-100 \%[1,5,17,18]$. However there are still some false negative results due to circumferential oedema and haemorrhage, a negative $\mathrm{CT}$ does not exclude the presence of a TBI $[7,14]$.

In our first case $\mathrm{CT}$ revealed only a right-sided pneumothorax. The patient had subcutaneous emphysema, and even after chest drainage she was still hypoxic, thus that was which strongly suggested the presence of TBI. In Case 2 respiratory distress, subcutaneous emphysema, blood clot in the larynx were found, and pneumothorax on both sides were managed by chest tubes. CT revealed pneumomediastinum, which together lead to a suspicion of TBI. In the third case contusion on the neck was discovered on physical examination. CT showed extremely spreading subcutaneous emphysema and pneumomediastinum as indirect signs, and a real direct sign of TBI was also revealed as the endotracheal tube's cuff was overinflated and presented external from the tracheal wall. All our three patients required bronchoscopic examination to confirm the diagnosis, and to identify the site of lacerations.

After CT an additional three-dimensional reconstruction or virtual bronchoscopy should be considered as a valuable help in diagnosis $[15,18]$. Oesophageal injuries are frequently associated, especially after direct trauma on neck, when trachea is smashed onto the oesophagus and cervical vertebral bodies. Contrast esophagogram can supplement the diagnosis and reveals a posterior tra- 
cheal wall rupture [8]. In case of a complete tracheal rupture, we should always search for concomitant injury of the oesophagus as well.

\section{Endoscopic examination}

Laryngoscopy and bronchoscopy are crucial part of diagnostic procedure. However, the CT has several advantages, these still remain the "gold standard" diagnostic tools. Laryngoscopy should be performed by otolaryngologist in cervical trauma or in suspicion of a laryngeal injury. It should be applied preferably with fibreoptic laryngo- or bronchoscopy through endotracheal tube. It is quick, easy and could be applied even in case of cervical spine injury [11]. All patients with suspicion of airway injury should undergo this examination as early as possible $[2,4,5,17]$. The most common findings include tear of the wall, blood clot filling the airway and collapsed lumen distal from the haematoma [1]. In some mild cases with simple mucosal laceration a small amount of blood could be the only sign for clinicians. If the endotracheal tube lays over the laceration part the lesion can be missed. For this reason, some of the patients are diagnosed only after extubation. If having a high suspicion of tracheal injury, it is better to deflate the tube's cuff and manipulate with the tube during bronchoscopy.

In Case 1 bronchoscopic examination revealed a rupture of the right main bronchus at the level of the carina. It was defined in Cardillo's classification [16] as a Level III/B laceration, and needed prompt surgical treatment, as having a high risk of developing mediastinitis. We did urgent operation and found a $4 \mathrm{~cm}$ long tear on the membranous trachea and a complete transection of the right main bronchus. In the second case bronchoscopy showed deformation in the bifurcation, but its lumen was intact, which was classified as Level III/A laceration and could be managed either surgical or non-surgical. During operation we found a longitudinal laceration separating the two main bronchi. In the last case CT showed an overinflated tube cuff and laryngoscopic examination confirmed the suggestion of a laceration at the level of the endotracheal tube's balloon. We found a completely transected trachea between the first and second tracheal rings. It was a Level IIIB laceration by Cardillo, or a Group V. rupture by Schaefer and Fuhrman's laryngotracheal classification method, and had urgent surgical indication.

\section{Emergency airway management}

Literary data consist that the most important priority is immediate intubation in patients suffering from TBI. Intravenous paralysis should be avoided, because transected airway is possibly attached and supported only by surrounding muscle. In addition an attempt of blindly intubation may lead to a false passage external from the airway, therefore intubation under the visualization of flexible bronchoscope is preferred $[1,2,9,12]$. It is important, that the tube should bypass the injured part and the cuff should be inflated distally to avoid further disrupt, even complete transection $[1,12,19]$. Low-tidal volume or low pressures may also prevent further disruption.

Tracheostomy is rarely necessary. It is recommended in patient with severe maxillofacial trauma, unstable fractures of the face, palpable deformity over the trachea, extremely oedema or haemorrhage. Other indications include severe oedema leading to obstruction, and inability to intubation. The tracheostomy is suggested to be caudal from the level of the injury. We should be aware that in case of a complete transection, the distal part of the trachea may slip into the mediastinum [11,17]. After tracheostomy, a definitive airway repair can be managed later.

Disruptions at the site of the bifurcation may require a doublelumen tube or selective endobronchial intubation [19].
In a difficult situation the surgeon can help with his finger in tube guidance through thoracotomy wound.

\section{Surgical management}

Treatment of airway injuries should be individualized at every patient, considering comorbidities, location of the lesion and clinical presentation [4]. After safe airway management and necessary diagnostic steps, the patient should be transferred to the operating room for reconstruction as soon as possible. The most important purposes of the surgery are 1) preventing airway obstruction, 2) avoiding spontaneous healing complications (such as stenosis), 3) avoiding mediastinitis 4) stop massive air leak and improve sufficient ventilation [1].

Independently of the lesion's site, a primary reconstruction should be attempted. Proximal part of the trachea is available through a low collar incision, which ensures a good exposure of vascular or oesophageal injuries. In middle-third tracheal injury a partial sternotomy over the manubrium with or without a collar incision provides a good view. We should keep it in mind, that in case of a complete transection in the cervical trachea, the distal part can easily retract into the mediastinum, leading to severe symptoms and making more difficult to approach it.

Distal third of the trachea, bifurcation and bronchi can be reached via a left or right thoracotomy in the 4-5TH intercostal space [1]. The access of the left main bronchus has some difficulties and seldom requires mobilisation of the aorta and division of the ligamentum arteriosum [2]. In some selected cases a median sternotomy can be an alternative approaching method. We mostly use a right sided thoracotomy if intrathoracic trachea, carina, right main bronchus or the proximal half of the left main stem bronchus injured.

For small transversal tears interrupted sutures are recommended, where the knots should lie outside the lumen to reduce the postoperative irritating and cough, and to avoid late stenosis. Longitudinal lacerations can be accomplished by continuous running suture. Larger disruption or complete transection requires refreshing on the damaged edges prior to primary end-to-end anastomosis $[1,13]$. Excessive airway mobilization should be avoided in order to prevent devascularisation, which may lead to late sequels, as dehiscence or stenosis. As far as possible primary repair of the carina should be performed and its resection should be avoided. If a resection is inevitable only an up to $3-4 \mathrm{~cm}$ long section of the airway allowed to be resected $[1,5,11,20]$.

If there is an extremely wide tissue damage and a primary repair cannot be performed a tracheostomy should be done. In case of laryngocricoid or both recurrent laryngeal nerves injury a protective tracheostomy is recommended $[1,11]$. The site of the tracheostomy should be as far as possible from tracheal restoration reducing the risk of dehiscence or stenosis. When resection is inevitable a tension-free anastomosis can be provided by blunt dissection of the avascular pretracheal plane. Flexion in the neck can be performed also for the purpose of a tension-free anastomosis for some days. For intrathoracic tension-free anastomosis, an opening on the pericardium or division of the inferior pulmonary ligaments can be performed. These techniques allow a $1-2 \mathrm{~cm}$ mobilization of the airway $[1,2,11,12]$.

In case of a severe airway disruption protective tissue flap (pleura, pericardium, mediastinal fat, muscle flap) can be used to cover the anastomosis or separate the oesophagus $[1,5,13]$. Severely distorted lung parenchyma requires lung resection occasionally.

All our three patients had primary anastomosis with 3-0 interrupted absorbable sutures without any resection. Two cases with lower airway injury had a pedicle intercostal muscle flap for covering, thus we successfully avoided postoperative dehiscence. Laryn- 
gotracheal laceration needed extended mobilisation of the trachea as the separated part were $3 \mathrm{~cm}$ apart from each other.

\section{Non-surgical management}

In some selected cases conservative treatment can be considered. Stated criterion include the followings: tear should be under $2 \mathrm{~cm}$ or one-third of the diameter, the patient should be clinically stable, breaths spontaneously or requires minimal ventilator support, and there should be no sign of mediastinitis or sepsis $[1,2,11,13]$.

According to Cardillo et al. [16] who classified airway lacerations, an incomplete rupture of the muscular wall (L. I, II) or simple complete wall rupture (L. III/A) can be managed non-operatively. Surgical approach is required when complete laceration associated with mediastinitis or coexistent oesophageal injury (L. III/B) or in case of separation between the two tracheal or bronchial parts.

Conservative treatment is mostly suggested in iatrogenic cases. These patients need antibiotic therapy and drainage. Previously reported, conservatively managed largest tracheal laceration was $4 \mathrm{~cm}$ long [2].

Self-expanding metallic stent (SEMS) can be used as alternative treatment in patients who have high surgical risk or severe comorbidities. This novel method is also useful in case of late stenosis caused by granulation tissue at the site of laceration [12].

For unstable patient with ventilatory failure extracorporeal membrane oxygenation (ECMO) is a valuable possibility, successfully applied by several authors [21,22,23,24,25].

\section{Postoperative management}

Postoperative earliest extubation is recommended to minimize the local trauma which is caused by the tube or elevated endotracheal pressure $[2,9,11,15]$. After isolated airway injury extubation can be safely performed in the operating room. If mechanical ventilation is necessary, the lowest pressure should be applied, and frequent pulmonary toilet is required to remove secretion [11]. Especially patients having vocal cord paralysis need aggressive pulmonary toilet, in which a mini tracheostomy seems to be helpful to allow a direct tracheal aspiration. In every case clinician should be convinced that the tube's cuff does not overlie onto the repaired site.

When early surgery can be managed a good long-term outcome is expected. It also depends on the severity of the associated injuries, developing septic condition, the necessity of intubation and intensive care unit support. Postoperative bronchoscopy should be performed after 7 to 10 days to ensure satisfactory healing and excluding stenosis [11].

Complications develop in up to $25,8 \%$ of the patients, which includes early anastomotic dehiscence, mediastinitis or 2-6 weeks later airway stenosis [1]. The incidence of stenosis and anastomotic dehiscence is 5\%-6\% [11]. These lead to recurrent pneumonia, bronchiectasis, or finally complete obstruction [2]. Some of these patients need an airway resection and reconstruction after 3 to 6 months. Injury of the larynx or recurrent laryngeal nerves may lead to phonation problems.

Initial diagnosis of TBI can be missed in $25 \%-68 \%$ of the patients [11]. This is due to mostly the serious associated injuries which obscure the symptoms of airway lesion. In other cases, the rupture might be small enough to cause only mild symptoms. The respiratory tract heals in 1-4 weeks after injury, but stricture may appear due to granulation tissue. This stenosis can cause obstruction in the airway, leading to consequential pneumonia, bronchiectasis or atelectasis [11].

\section{Conclusions}

An algorithmic approach for surgical or non-surgical management of thoracic airway injury is offered. The most important steps to prompt primary management according to the contemporary literature are summarized (Figs. 6, 7).

When patient presents on emergency department the first step is to consider whether she or he had a trauma which can lead to airway injury (previous flexion-extension or compression forces, sign of an elevated seat-belt, blood in the vomit or sputum). The most important symptoms we should search for on physical examination include dyspnoea, respiratory distress, subcutaneous emphysema, suspected pneumothorax and contusion over the neck and thorax. Stable patient should be referred for prompt MDCT examination, and if there are signs of tracheobronchial injury (pneumomediastinum, spreading subcutaneous emphysema, persistent pneumothorax despite of tube thoracostomy), then laryngoscopy or bronchoscopy should be immediately done to localise the site of the laceration. At any time when it is necessary an intubation guiding by fiberscope is a good choice, at least when laryngoscopic/bronchoscopic examination is applied. Here we have an opportunity to perform esophagoscopy if a posterior wall disruption is suspicious and the diagnosis is not clear. If airway injury is confirmed, we should transfer the patient to the operation room as soon as possible. In some selected cases when special conditions are fulfilled conservative therapy can be a valuable alternative choice. These conditions are: tear should be under $2 \mathrm{~cm}$ or one-third of the diameter, the patient should be clinically stable, breaths spontaneously or requires minimal ventilator support, and there should be no sign of mediastinitis or sepsis. Conservative therapy should be disestablished and surgical management should be done at any time when healing process or the patient's condition is not satisfying.

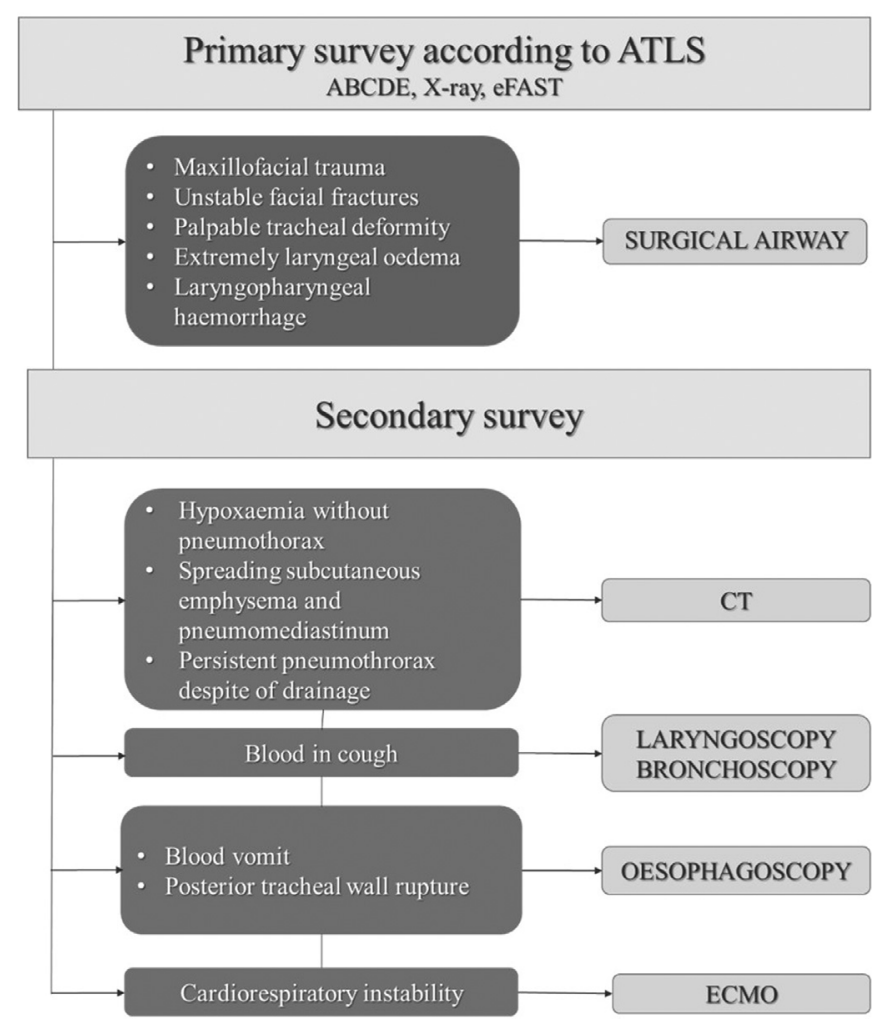

Fig. 6. Diagnostic and emergency intervention flowchart. 

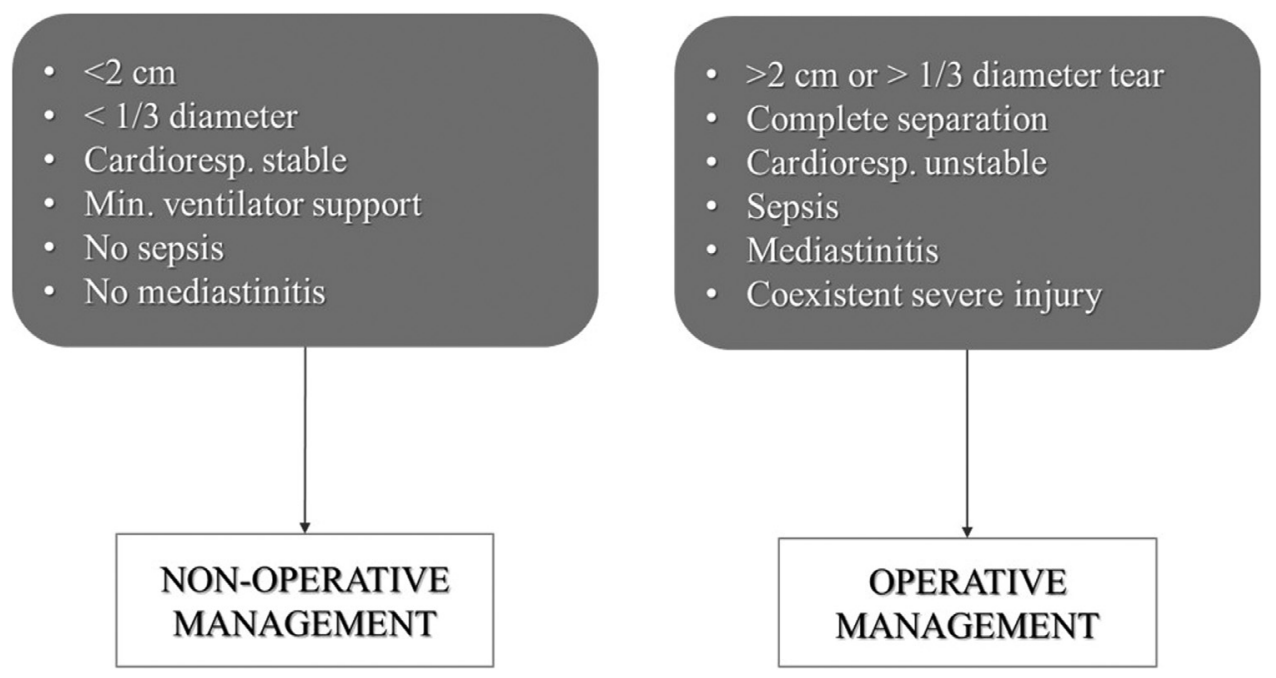

Fig. 7. Treatment flowchart of TBI.

\section{Declaration of Competing Interest}

All the authors declare that they have no conflict of interest or funding.

\section{Supplementary materials}

Supplementary material associated with this article can be found, in the online version, at doi:10.1016/j.injury.2020.07.026.

\section{References}

[1] Prokakis C, Koletsis EN, Dedeilias P, Fligou F, Filos K, Dougenis D. Airway trauma: a review on epidemiology, mechanisms of injury, diagnosis and treatment. J Cardiothorac Surg 2014;9:117 Jun 30.

[2] Kuzdzal J, Asamura H, Detterbeck F, Goldstraw P, Lerut A, Thomas P, et al.. ESTS textbook of thoracic surgery vol 2. Cracow: Medycyna Praktyczna; 2015. p. 937-43.

[3] Bertelsen S, Howitz P. Injuries of the trachea and bronchi. Thorax 1972;27(2):188-94 Mar.

[4] Gallagher JJ. Management of blunt pulmonary injury. AACN Adv Crit Care 2014;25(4):375-86 Decquiz 387-8.

[5] Welter S. Repair of tracheobronchial injuries. Thorac Surg Clin 2014;24(1):41-50 Feb.

[6] Dominguez E, Torre CDL, Sánchez AV, Hernandez F, Ortiz R, Moreno AMA, et al. Severe Tracheobronchial Injuries: our Experience. Eur J Pediatr Surg 2015;25(01):71-6 Feb.

[7] Cheng J, Cooper M, Tracy E. Clinical considerations for blunt laryngotracheal trauma in children. J Pediatr Surg 2017;52(5):874-80 May.

[8] Book. ATLS advanced trauma life support, student course manual. 10th Edition. American College of Surgeons; 2018.

[9] van Roozendaal LM, van Gool MH, Sprooten RTM, Maesen BAE, Poeze M, Hulsewé KWE, et al. Surgical treatment of bronchial rupture in blunt chest trauma: a review of literature. J Thorac Dis 2018;10(9):5576-83 Sep.

[10] Kirsh MM, Orringer MB, Behrendt DM, Sloan H. Management of tracheobronchial disruption secondary to nonpenetrating trauma. Ann Thorac Surg 1976;22(1):93-101.
[11] Altinok T, Can A. Management of Tracheobronchial Injuries. Eurasian J Med 2014;46(3):209-15 Oct.

[12] Shemmeri E, Vallières E. Blunt Tracheobronchial Trauma. Thorac Surg Clin 2018;28(3):429-34 Aug 1.

[13] Zhao Z, Zhang T, Yin X, Zhao J, Li X, Zhou Y. Update on the diagnosis and treatment of tracheal and bronchial injury. J Thorac Dis 2017:9(1):E50-6 Jan.

[14] Santiago-Rosado LM, Sigmon DF, Lewison CS. Tracheal Trauma. [Updated 2020 Apr 28]. In: StatPearls [Internet]. Treasure Island (FL): StatPearls Publishing; 2020 Jan. Available from: https://www.ncbi.nlm.nih.gov/books/NBK500015/

[15] Alassal MA, Ibrahim BM, Elsadeck N. Traumatic intrathoracic tracheobronchial injuries: a study of 78 cases. Asian Cardiovasc Thorac Ann 2014;22(7):816-23 Sep 1.

[16] Cardillo G, Carbone L, Carleo F, Batzella S, Jacono RD, Lucantoni G, et al. Tracheal lacerations after endotracheal intubation: a proposed morphological classification to guide non-surgical treatment. Eur J Cardiothorac Surg 2010:37(3):581-7 Mar:

[17] Bagga B, Kumar A, Chahal A, Gamanagatti S, Kumar S. Traumatic Airway Injuries: role of Imaging. Curr Probl Diagn Radiol. 2020 01;49(1):48-53.

[18] Newbury A, Dorfman JD, Lo HS. Imaging and Management of Thoracic Trauma. Semin Ultrasound CT MR 2018;39(4):347-54.

[19] Elgendy H, Jilani T. Successful anesthetic management in a child after traumatic rupture of left main bronchus by a single-lumen cuffed-endotracheal tube. Ann Card Anaesth 2014;17(4):292 Jan 10.

[20] Dennis BM, Bellister SA, Guillamondegui OD. Thoracic Trauma. Surg Clin North Am 2017;97(5):1047-64 Oct.

[21] Biancosino C, Krüger M, Kühn C, Zinne N, Wilhelmi M, Zeckey C, et al. First Successful Surgical Reconstruction of Bilateral Transected Main Bronchi With Extracorporeal Membrane Oxygenation Support. Ann Thorac Surg 2016;102(2):e135-7.

[22] Ryu KM, Chang SW. Heparin-free extracorporeal membrane oxygenation in a patient with severe pulmonary contusions and bronchial disruption. Clin Exp Emerg Med 2018;5(3):204-7 Apr 30.

[23] Hamilton EC, Lazar D, Tsao K, Cox C, Austin MT. Pediatric tracheobronchial injury after blunt trauma. J Trauma Acute Care Surg 2017:83(3):554-6.

[24] Suh JW, Shin HJ, Lee CY, Song SH, Narm KS, Lee JG. Surgical Repair of a Traumatic Tracheobronchial Injury in a Pediatric Patient Assisted with Venoarterial Extracorporeal Membrane Oxygenation. Korean J Thorac Cardiovasc Surg 2017:50(5):403-6 Oct.

[25] Noh D, Lee C, Hwang JJ, Cho HM. Concomitant Avulsion Injury of the Subclavian Vessels and the Main Bronchus Caused by Blunt Trauma. Korean J Thorac Cardiovasc Surg 2018;51(2):153-5 Apr. 